\title{
Melatonin modulation of crosstalk among malignant epithelial, endothelial and adipose cells in breast cancer (Review)
}

\author{
SAMUEL COS, VIRGINIA ÁLVAREZ-GARCÍA, ALICIA GONZÁLEZ, \\ CAROLINA ALONSO-GONZÁLEZ and CARLOS MARTÍNEZ-CAMPA \\ Department of Physiology and Pharmacology, School of Medicine, \\ University of Cantabria and Valdecilla Research Institute, Santander 39011, Spain
}

Received October 28, 2013; Accepted May 13, 2014

DOI: $10.3892 / \mathrm{ol} .2014 .2203$

\begin{abstract}
Melatonin, the main secretory product of the pineal gland, is an oncostatic agent that reduces the growth and development of various types of tumors, particularly mammary tumors whose growth is dependent on estrogens. Previous in vivo and in vitro studies point to the hypothesis that melatonin interplays with estrogen signaling pathways at three different levels: i) an indirect mechanism, by interfering with the hypothalamic-pituitary-reproductive axis in such way that the level of plasma estrogens synthesized by the gonadal glands are downregulated; ii) a direct mechanism of the pineal gland at the cell cancer level, disrupting the activation of estradiol receptors, therefore behaving as a selective estrogen receptor modulator; and iii) by regulating the enzymes involved in the biosynthesis of estrogens in other tissues, thus behaving as a selective estrogen enzyme modulator. The intratumoral metabolism and synthesis of estrogens, as a result of the interactions of various enzymes, is more important than blood uptake to maintain mammary gland estrogen levels in menopausal females. Additionally, estrogens are considered to play an important role in the pathogenesis and development of hormone-dependent breast carcinoma. Paracrine interactions among malignant epithelial cells and proximal adipose and endothelial cells, through cytokines and growth factors produced by breast tumor cells, modulate estrogen production at the mammary tumor level and, as a consequence, the genesis and development of mammary tumors. The aim of the present review is to summarize the recent findings describing the mechanisms by which melatonin is able to modulate the crosstalk
\end{abstract}

Correspondence to: Dr Samuel Cos, Department of Physiology and Pharmacology, School of Medicine, University of Cantabria and Valdecilla Research Institute, Cardenal Herrera Oria s/n, Santander 39011, Spain

E-mail: coss@unican.es; samuel.cos@unican.es

Key words: melatonin, pineal gland, endothelial cell, fibroblast, aromatase, MCF-7 among malignant epithelial, endothelial and adipose cells in breast cancer.

\section{Contents}

1. Introduction

2. Local synthesis of estrogens in breast cancer epithelial cells and melatonin

3. Local synthesis of estrogens in peritumoral fibroblasts and melatonin

4. Local synthesis of estrogens in peritumoral endothelial cells and melatonin

5. Conclusions

\section{Introduction}

The oncostatic effects of melatonin are particularly relevant to hormone-dependent tumors (1-5). Of these neoplasias, the most deeply studied have been mammary adenocarcinomas. Based on the role of the pineal gland in inhibiting gonadal maturation and sex hormone secretion in mammals, Cohen et al (1978) introduced the hypothesis that a decrease in pineal function decreases melatonin levels and induces a relative 'hyperestrogenism', which underlies the development of breast cancer (6). Since then, there has been evidence supporting the theory that the antitumor actions of melatonin in hormone-dependent tumors are mainly based on the antiestrogenic properties of melatonin $(5,7)$.

The oncostatic effects of melatonin in hormone-dependent breast cancer were firstly explained by indirect neuroendocrine mechanisms, such as the downregulation of the neuroendocrine reproductive axis by melatonin, and the consequent reduction of estrogenic hormones responsible for the normal and pathological growth of the mammary gland (8). In addition, it has also been demonstrated that melatonin may directly interfere with the activation of the estrogen receptor and counteract the effects of estrogens at the tumor cell level, thus behaving as a selective estrogen receptor modulator (7,9-11). In more recent years, a third neuroendocrine mechanism has been described in which melatonin is able to reduce the estrogen-mediated development of breast cancer, involving 
the regulation of certain enzymes responsible for the local synthesis of estrogens, thus behaving as a selective estrogen enzyme modulator (12-15).

\section{Local synthesis of estrogens in breast cancer epithelial cells and melatonin}

The intratumoral metabolism and synthesis of estrogens, as a result of the interactions of various enzymes, is considered to play an important role in the pathogenesis and development of hormone-dependent breast carcinoma (16-19). In breast cancer, particularly that of postmenopausal women, estrogens are synthesized in the mammary tissue by transformation either from androgen precursors, mainly of adrenal origin, or from biologically inactive estrogens. Breast carcinoma epithelial cells contain all the enzymes necessary for the local synthesis of estrogens (Fig. 1). One of the major pathways involved in the synthesis of estrogens in breast cancer cells is the aromatase pathway, which transforms androgens into estrogens (20). Aromatase activity and expression is markedly higher in breast cancer tissue than in normal mammary tissue $(21,22)$. The second pathway involved in estrogen formation is the sulfatase pathway, which converts estrogen sulfates into estrone and estradiol $(18,19,22)$. The final step of steroidogenesis in peripheral tissues is the conversion of the weak estrone to the potent biologically active estradiol by the action of the $17 \beta$-hydroxysteroid dehydrogenase activity type $1(17 \beta-H S D 1)(18,19)$. In breast cancer tissue, estrogen sulfotransferase is also present, which converts estrogens into estrogen sulfates. Since the sulfo-conjugated estrogens are the biologically inactive forms of the estrogens, another possible way to control the tissular concentration of active estradiol is to identify new ways to stimulate the enzymes involved in the sulfate formation $(19,22)$.

In normal breast tissue, there is a high concentration of circulating inactive steroids, which are the major precursor substrates of local estrogen production, mainly estrone. In this tissue, the estrogen sulfotransferase activity and expression, the enzyme that inactivates estrone and $17 \beta$-estradiol, tend to be increased. However, in breast carcinoma tissue, aromatase (which converts androgens into estrogens), sulfatase (which hydrolyzes the estrone sulfates to estrone) and 17 $\beta$-HSD1 (which converts the estrone to the potent $17 \beta$-estradiol) tend to be overexpressed, whereas the expression of estrogen sulfotransferase is frequently decreased, which may result in the accumulation of 17ß-estradiol in breast cancer tissues (Fig. 2). At the tumor cell level, melatonin decreases the activity and expression of aromatase, sulfatase and 17 $\beta$-HSD1, and increases the activity and expression of estrogen sulfotransferase $(12,13,23,24)$. Melatonin tends to modify the activity and expression of the enzymes involved in the local synthesis of estrogens, causing them to be similar to the expression of enzymes in the mammary normal tissue, and may thus protect mammary tissue from excessive estrogenic effects (Fig. 2).

Regulation of aromatase expression in human tissues is relatively complex, involving alternative promoter sites that provide tissue-specific control. In the normal breast, the mammary adipose tissue maintains low levels of aromatase expression almost exclusively via promoter I.4 (25). However, in mammary cancer, both in malignant epithelial cells and

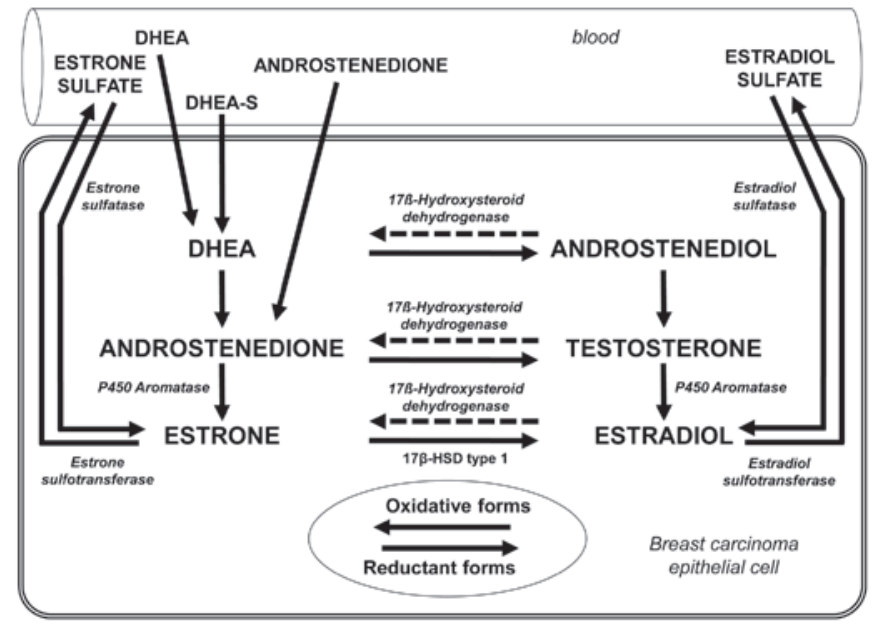

Figure 1. Enzymatic mechanisms involved in the local production of estrogens in human breast carcinoma tissues. DHEA, dehydroepiandrosterone; DHEA-S, DHEA sulfate; $17 \beta$-HSD type 1, 17 $\beta$-hydroxysteroid dehydrogenase type 1 .

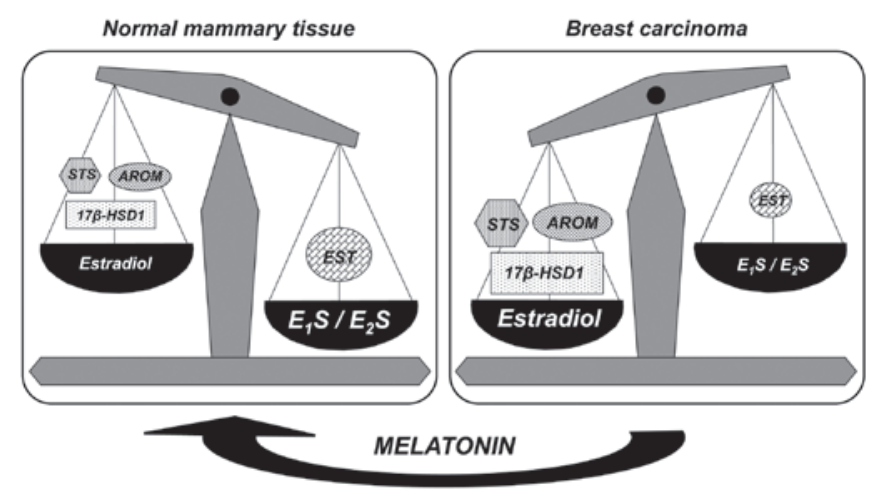

Figure 2. Expression of enzymes associated with the local production of estrogens in human normal mammary tissue and breast carcinoma tissue. In the breast carcinoma tissue, STS, AROM and 17 $\beta$-HSD1 tend to be overexpressed, while EST is decreased. Melatonin decreases the expression of AROM, STS and 17 $\beta$-HSD1, and increases the EST expression. Thus, melatonin tends to modify the expression of the enzymes involved in the local synthesis of estrogens, causing it to be similar to the expression of enzymes in the mammary normal tissue. Figure modified from Cos et al (23). STS, sulfatase; AROM, aromatase; 17 $\beta$-HSD1, 17 $\beta$-hydroxysteroid dehydrogenase type 1; EST, estrogen sulfotransferase; $\mathrm{E}_{1} \mathrm{~S}$, estrone sulfate; $\mathrm{E}_{2} \mathrm{~S}$, estradiol sulfate.

fibroblasts, the expression of aromatase is increased via activation of promoters II and I.3, which are regulated by cyclic adenosine monophosphate (cAMP) and factors that regulate cAMP levels (26). Prostaglandin $\mathrm{E}_{2}\left(\mathrm{PGE}_{2}\right)$ is an important regulator of aromatase gene expression via promoters II and I.3 (25-28). The formation of $\mathrm{PGE}_{2}$ occurs through the activity of the cyclooxygenases (COXs), rate-limiting enzymes that catalyze the conversion of arachidonic acid to prostaglandins. Promoter I.3 and II are considered to be the major promoters driving aromatase expression in breast cancer and surrounding adipose tissue. One of the mechanisms through which melatonin modulates aromatase enzyme in breast tumor cells is through its downregulatory action on the expression of COX enzymes, COX-1 and COX-2, which decrease the levels of $\mathrm{PGE}_{2}$. Lower levels of $\mathrm{PGE}_{2}$ result in decreased intracellular levels of cAMP, which in turn diminish the activation 
of promoters I.3 and II, and result in decreased aromatase expression $(29,30)$.

In addition, the antiaromatase and antisulfatase effects of melatonin have also been shown in cancer cell types other than breast cancer cells. In glioblastoma cells, which express estrogen receptors and have the ability to synthesize estrogens, melatonin also reduces the local production of estrogens by decreasing the activity of aromatase, sulfatase and 17 $\beta$-HSD1, and downregulating aromatase, sulfatase and 17 $\beta$-HSD1 mRNA steady state levels $(31,32)$.

This melatonin modulatory effect on the aromatase and sulfatase enzymes, at the tumor cell level, has also been described in vivo, in rats bearing 7,12-dimethylbenzanthracene-induced mammary tumors. The growth of these mammary tumors is estrogen-dependent and ovariectomy significantly reduces both the size and number of tumors, while the administration of testosterone or estrone sulfate to ovariectomized animals is able to maintain the tumor growth at the same level as the control (uncastrated) animals. The stimulatory effects of tumor development induced by testosterone (which depends on the local synthesis of estrogens from androgens), due to the aromatase action, or estrone sulfate (which depends on the estrogens locally formed by the action of the sulfatase enzyme on the biologically inactive estrogens), are suppressed by the administration of melatonin. Tumors from animals treated with melatonin have the lowest microsomal aromatase and sulfatase activity $(14,33)$.

\section{Local synthesis of estrogens in peritumoral fibroblasts and melatonin}

In breast tumors, the majority of aromatase and sulfatase activity and expression, the two principal pathways of synthesis of estrogens, are found in the fibroblast component of the adipose tissue and in vascular endothelial cells. The local biosynthesis of estrogens in breast cancer depends on paracrine interactions between malignant epithelial cells and proximal fibroblasts and vascular endothelial cells. Malignant epithelial cells secrete cytokines, including tumor necrosis factor $\alpha$ (TNF- $\alpha$ ), interleukin 6 (IL-6) and IL-11, which are upregulated by estrogens. These cytokines inhibit the differentiation of surrounding fibroblasts into mature adipocytes, through the selective inhibition of expression of peroxisome proliferator-activated receptor $\gamma(\operatorname{PPAR} \gamma)$ and CCAAT/enhancer binding protein $\alpha$ $(\mathrm{C} / \mathrm{EBP} \alpha)$, and also stimulate aromatase expression in these undifferentiated fibroblasts (Fig. 3) (19,34,35). This biological phenomenon is commonly known as the desmoplastic reaction or the accumulation of undifferentiated fibroblasts with high aromatase activity surrounding malignant epithelial cells. Tumor cells also secrete other factors, such as $\mathrm{PGE}_{2}$, which stimulate aromatase activity and expression in these undifferentiated fibroblasts, as well as upregulating antiadipogenic cytokines.

3T3-L1 is a fibroblast cell line that is initially fibroblastic but which, under appropriate conditions, differentiates into adipocytes (36). Melatonin treatment during the preadipocyte differentiation enhances the adipogenesis, and higher doses of melatonin induce more extensive deposits of lipid droplets and also induce a $\sim 50 \%$ reduction in the aromatase activity of the cells, two indicators of adipogenic differentiation. It

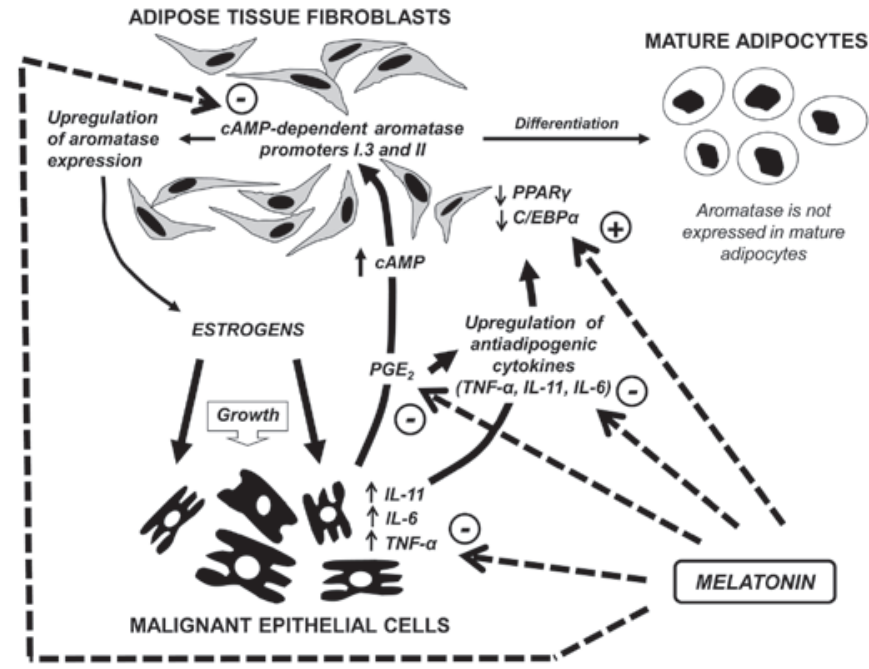

Figure 3. Epithelial-stromal interactions in breast tumors inhibit adipogenic differentiation and enhance estrogen formation by increasing the aromatase activity of the undifferentiated fibroblasts. All these actions are mediated by cytokines, such as TNF- $\alpha$, IL- 6 and IL-11, produced by malignant epithelial cells. Melatonin reduces the formation of undifferentiated fibroblasts surrounding malignant epithelial cells by stimulating the differentiation of fibroblasts to mature adipocytes and adipogenesis, and by decreasing the aromatase activity of the fibroblasts and adipocytes through a downregulatory action on the expression of antiadipogenic cytokines, which decreases the levels of these cytokines. Lower levels of TNF- $\alpha$, IL- 6 and IL-11 stimulate the differentiation of fibroblasts and decrease the aromatase activity and expression. Melatonin also decreases the production of $\mathrm{PGE}_{2}$ by malignant cells, which upregulates aromatase expression both in the tumor itself and in the surrounding adipose tissue, and enhances the production of IL-11 by tumor cells. Figure modified from Álvarez-García et al (39) and Cos et al (23). TNF- $\alpha$, tumor necrosis factor- $\alpha$; IL, interleukin.

has been demonstrated that melatonin significantly increases the expression of PPAR $\gamma$ and $\mathrm{C} / \mathrm{EBP} \alpha$, the two main regulators of terminal adipogenesis (37). An approach to simulate in vitro the situation occurring in the mammary tumor is to use cocultures of malignant epithelial cells with fibroblasts or endothelial cells. The presence of malignant epithelial cells in the cocultures inhibits the differentiation of preadipocytes to adipocytes and reduces the intracytoplasmic triglyceride accumulation, an indicator of adipogenic differentiation. The presence of malignant cells also stimulates the aromatase activity in the fibroblasts. Melatonin counteracts the inhibitory effect on adipocyte differentiation induced by malignant epithelial cells, and also counteracts the stimulatory effect of the presence of breast cancer cells on aromatase activity in fibroblasts (37-39).

The levels of antiadipogenic cytokines, TNF- $\alpha$, IL- 6 and IL-11, in the coculture media are 10-fold higher than those found in the culture of fibroblasts alone, since epithelial malignant cells, in the presence of fibroblasts, secrete these cytokines with the aim to inhibit the differentiation of preadipocytes into adipocytes and to accumulate undifferentiated fibroblasts with high aromatase activity around malignant epithelial cells. The addition of melatonin to the cocultures decreases the concentrations of cytokines in the media and counteracts the stimulatory effect induced by the presence of malignant cells on the cytokines levels. Melatonin also induces a reduction in the TNF- $\alpha$, IL- 6 and IL-11 mRNA expression in breast cancer epithelial cells and fibroblasts (Fig. 3). The 
addition of luzindole, a melatonin receptor antagonist, prevents this inhibitory effect of melatonin on cytokines expression, indicating that melatonin acts through known melatonin receptor-mediated mechanisms (39).

In summary, melatonin may reduce the level of undifferentiated fibroblasts surrounding malignant epithelial cells by stimulating the differentiation of fibroblasts to mature adipocytes and adipogenesis, and by decreasing the aromatase activity of the fibroblasts through a downregulatory action on the expression of antiadipogenic cytokines, which decreases the levels of these cytokines. Lower levels of TNF- $\alpha$, IL-6 and IL-11 allow the differentiation of fibroblasts, as well as decreasing the aromatase activity and expression. Melatonin also decreases the production of $\mathrm{PGE}_{2}$ by malignant cells, which downregulates aromatase expression and cytokine production in the tumor itself and in the surrounding adipose tissue. Lower levels of aromatase lead to lower levels of estrogens, resulting in decreased growth and development of the breast tumor (Fig. 3).

\section{Local synthesis of estrogens in peritumoral endothelial cells and melatonin}

Endothelial cells also represent a critical cellular element in the tumor microenvironment, which play a crucial role in the growth and progression of breast tumors. They are another source of estrogens, as they also express aromatase $(40,41)$. Promoter I.7 is a novel breast cancer-associated aromatase promoter mainly active in vascular endothelial cells, and is upregulated in breast cancer tissue (42). Excessive aromatase expression via promoters I.3, II and I.7, and consequent increase in estrogen biosynthesis in malignant epithelial cells, undifferentiated adipose fibroblasts and adjacent endothelial cells contribute to the development and progression of breast cancer. In addition, endothelial cells provide structural and biochemical support for tumor growth and progression of cancer through control of angiogenesis. Vascular endothelial growth factor (VEGF) secreted by breast cancer cells is essential for the expansion of breast cancer and may function in both paracrine and autocrine manners to promote the proliferation, growth, survival and migration of endothelial cells $(43,44)$.

In endothelial cells, melatonin decreases the aromatase activity and expression mainly by inducing a significant downregulation in aromatase expression specifically driven by promoter I.7, the major promoter directing aromatase expression in endothelial cells (Fig. 4) (45).

VEGF, a major regulator of endothelial growth, added to endothelial cell cultures stimulates the proliferation of these cells and melatonin counteracts this effect (46). Melatonin reduces VEGF mRNA expression in human breast cancer (MCF-7) cells and also reduces VEGF levels in cell culture media of malignant epithelial cells (Fig. 4). Cocultures of breast malignant epithelial cells and endothelial cells is an approach to simulate in vitro the paracrine interaction between these cells in the mammary tumors. The presence of malignant epithelial cells in the cocultures is able to stimulate the endothelial cell proliferation and increase the VEGF levels in the culture media. Melatonin counteracts the stimulatory effects on endothelial cell proliferation and on VEGF protein levels in the coculture media. The changes in endothelial cell

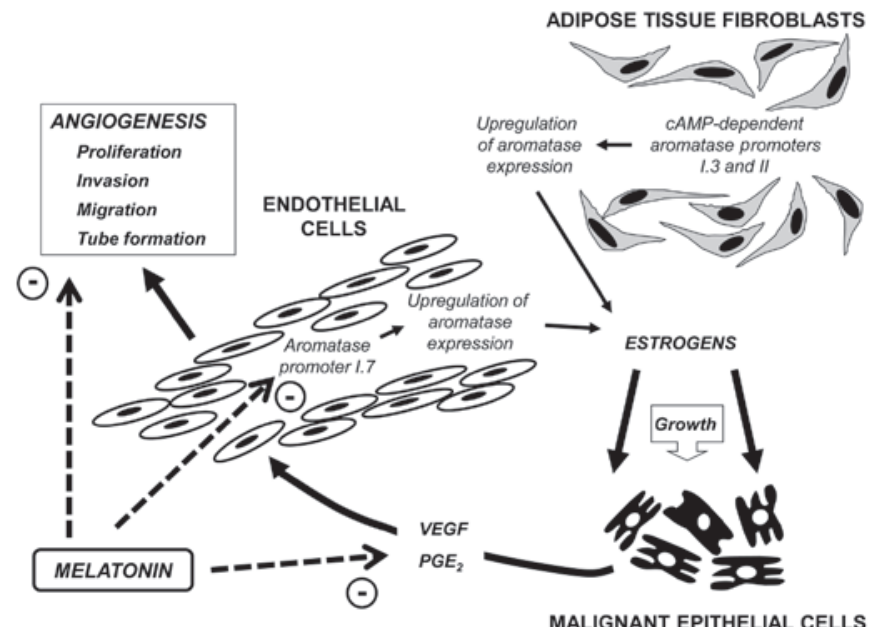

Figure 4. Role of melatonin in the paracrine interactions that occur between malignant epithelial cells and proximal endothelial cells. Melatonin may be important in reducing endothelial cell proliferation, invasion, migration and tube formation, through a downregulatory action on VEGF and $\mathrm{PGE}_{2}$. $\mathrm{PGE}_{2}$ induced by VEGF may directly promote angiogenesis and melatonin through its downregulatory action on the expression of cyclooxygenase enzymes, decreasing the levels of $\mathrm{PGE}_{2}$ and reducing the levels of angiogenesis. Melatonin also inhibits aromatase activity and expression in endothelial cells by regulating the gene expression of specific aromatase promoter region I.7, thereby reducing the local production of estrogens. VEGF, vascular endothelial growth factor; $\mathrm{PGE}_{2}$, prostaglandin $\mathrm{E}_{2}$.

proliferation induced by melatonin are mediated by an inhibition of the synthesis of VEGF in malignant epithelial cells. Conditioned media from malignant cells stimulate endothelial cell proliferation, and this effect is significantly counteracted by anti-VEGF and melatonin (46).

All these findings suggest that melatonin may play a role in the paracrine interactions between malignant epithelial cells and proximal endothelial cells, through a downregulatory action on VEGF expression in human breast cancer cells, which decreases the levels of VEGF surrounding endothelial cells. Lower levels of VEGF may be important in reducing the number of estrogen-producing cells proximal to malignant cells, as well as in decreasing tumoral angiogenesis. Antiangiogenic activity of melatonin against the pro-angiogenic effects of breast cancer cells has also been described (47). Recently, it has been demonstrated that melatonin has effects on different steps of the angiogenic process in endothelial cell cultures (47). Melatonin strongly inhibits the proliferation of endothelial cells and counteracts the stimulatory effect induced by estradiol. In Transwell assays, melatonin has been identified to reduce the number of endothelial cells that invaded through a basement membrane in response to VEGF. Endothelial cell migration is essential for the formation of new blood vessels during neo-angiogenesis. Melatonin treatment strongly inhibits the migration of endothelial cells in wound-healing assays. Another important step during neo-angiogenesis is the formation of tubes by endothelial cells. It is established that VEGF increases the formation of a branching network of tubes. Melatonin disrupts the tube formation and counteracts the VEGF-stimulated tubular network formation by endothelial cells. In addition, conditioned media collected from breast cancer cells are angiogenically active and stimulate tubule length formation. This effect is significantly counteracted by 
the addition of either anti-VEGF antibody or melatonin, which suggests that the melatonin-induced decrease of capillary structure formation stimulated by conditioned media from MCF-7 cells may occur as a result of inhibition of VEGF activity (47).

Melatonin may play a role in the paracrine interactions that take place between malignant epithelial cells and proximal endothelial cells, acting by different mechanisms. On one hand, melatonin exerts antiangiogenic effects and may be important in reducing endothelial cell proliferation, invasion, migration and tube formation, through a downregulatory action on VEGF and $\mathrm{PGE}_{2}$ (Fig. 4). $\mathrm{PGE}_{2}$ synthesis induced by VEGF may directly promote angiogenesis and melatonin through its downregulatory action on the expression of COX enzymes, which decrease the levels of $\mathrm{PGE}_{2}$ and reduce angiogenesis. On the other hand, melatonin inhibits aromatase activity and expression in endothelial cells by regulating gene expression of specific aromatase promoter regions, thereby reducing the local production of estrogens (Fig. 4).

\section{Conclusions}

Severallines of evidence highlight the contribution of the tumor microenvironment to its growth and maintenance. Cells immediately adjacent to the tumor are not only passive structural support but also active elements in tumor progression. Among the numerous different cell types surrounding breast cancer cells, the most abundant are those that compose mammary adipose tissue. Ninety percent of these resident cells of adipose tissue are fibroblasts, the precursors of mature adipocytes, and $7 \%$ are endothelial cells (35). Epithelial-stromal interactions in breast tumors inhibit adipogenic differentiation and enhance estrogen formation by increasing the aromatase activity of the undifferentiated fibroblasts. All these actions are mediated by cytokines, such as TNF- $\alpha$, IL-11 and IL-6, produced by malignant epithelial cells. Melatonin may reduce the formation of undifferentiated fibroblasts surrounding malignant epithelial cells by stimulating the differentiation of fibroblasts to mature adipocytes and adipogenesis, and by decreasing the aromatase activity of the fibroblasts and adipocytes through a downregulatory action on the expression of antiadipogenic cytokines, which decrease the levels of these cytokines. Lower levels of TNF- $\alpha$, IL- 6 and IL-11 stimulate the differentiation of fibroblasts and decrease the aromatase activity and expression. Melatonin also decreases the production of $\mathrm{PGE}_{2}$ by malignant cells, which upregulates aromatase expression both in the tumor itself and in the surrounding adipose tissue and enhances the production of IL-11 by tumor cells. Endothelial cells also produce estrogens from androgens precursors. Melatonin decreases the activation of promoter I.7 and results in decreased aromatase expression. In addition, melatonin reduces endothelial cell proliferation, invasion, migration and tube formation, through a downregulatory action on VEGF. This melatonin modulation of epithelial-stromal interactions favors lower numbers of undifferentiated fibroblasts, angiogenesis and reduced local estrogen concentrations in breast tumors.

Melatonin may play a role in the paracrine interactions that occur between malignant epithelial cells and proximal adipose and endothelial cells, through a downregulatory action on cytokines and growth factors produced by breast tumor cells. The actions of melatonin described in the present review involve antiproliferative, antiaromatase and antiangiogenic effects, and suggest that melatonin may potentially be beneficial as an anticancer drug in the prevention and treatment of estrogen-dependent mammary tumors. Therefore, this creates interesting possibilities for the clinical applications of melatonin in breast cancer.

\section{Acknowledgements}

This study was supported by grants from the Spanish Ministry of Science and Innovation (SAF2010-19579) and the Valdecilla Research Institute (APG-09-GC6).

\section{References}

1. Cos S and Sánchez-Barceló EJ: Melatonin and mammary pathological growth. Front Neuroendocrinol 21: 133-170, 2000

2. Cos S and Sánchez-Barceló EJ: Melatonin, experimental basis for a possible application in breast cancer prevention and treatment. Histol Histopathol 15: 637-647, 2000.

3. Blask DE, Sauer LA and Dauchy RT: Melatonin as a chronobiotic/anticancer agent: cellular, biochemical and molecular mechanisms of action and their implications for circadian-based cancer therapy. Curr Topics Med Chem 2: 113-132, 2002.

4. Sánchez-Barceló EJ, Cos S, Fernández R and Mediavilla MD: Melatonin and mammary cancer: a short review. Endocr Relat Cancer 10: 153-159, 2003.

5. Sánchez-Barceló EJ, Cos S, Mediavilla MD, Martínez-Campa CM, González A and Alonso-González C: Melatonin-estrogen interactions in breast cancer. J Pineal Res 38: 217-222, 2005.

6. Cohen M, Lippman M and Chabner B: Role of pineal gland in aetiology and treatment of breast cancer. Lancet 2: 814-816, 1978.

7. Cos S, González A, Martínez-Campa C, Mediavilla MD, Alonso-GonzálezC and Sánchez-Barceló EJ: Estrogen-signaling pathway: a link between breast cancer and melatonin oncostatic actions. Cancer Detect Prev 30: 118-128, 2006.

8. Reiter RJ: The pineal and its hormones in the control of reproduction in mammals. Endocr Rev 1: 109-131, 1980.

9. Molis TM, Spriggs LL and Hill SM: Modulation of estrogen receptor mRNA expression by melatonin in MCF-7 human breast cancer cells. Mol Endocrinol 8: 1681-1690, 1994.

10. Cos S, Blask DE, Lemus-Wilson A and Hill SM: Effects of melatonin on the cell cycle kinetics and estrogen rescue of MCF-7 human breast cancer cells in culture. J Pineal Res 10: 36-42, 1991.

11. Hill SM, Spriggs LL, Simon MA, Muraoka H and Blask DE: The growth inhibitory action of melatonin on human breast cancer cells is linked to the estrogen response system. Cancer Lett 64: 249-256, 1992.

12. Cos S, Martínez-Campa C, Mediavilla MD and Sánchez-Barceló EJ: Melatonin modulates aromatase activity in MCF-7 human breast cancer cells. J Pineal Res 38: 136-142, 2005.

13. González A, Martínez-Campa C, Mediavilla MD, et al: Effects of MT1 melatonin receptor overexpression on the aromatase-suppressive effect of melatonin in MCF-7 human breast cancer cells. Oncol Rep 17: 947-955, 2007.

14. Cos S, González A, Güezmes A, Mediavilla MD, Martínez-Campa C, Alonso-González C and Sánchez-Barceló EJ: Melatonin inhibits the growth of DMBA-induced mammary tumors by decreasing the local biosynthesis of estrogens through the modulation of aromatase activity. Int J Cancer 118: 274-278, 2006.

15. Martínez-Campa C, González A, Mediavilla MD, Alonso-González C, Sánchez-Barceló EJ and Cos S: Melatonin enhances the inhibitory effect of aminoglutethimide on aromatase activity in MCF-7 human breast cancer cells. Breast Cancer Res Treat 94: 249-254, 2005.

16. Simpson ER: Role of aromatase in sex steroid action. J Mol Endocrinol 25: 149-156, 2000. 
17. van Landeghem AA, Poortman J, Nabuurs M and Thijssen JH: Endogenous concentration and subcellular distribution of estrogens in normal and malignant human breast tissue. Cancer Res 45: 2900-2906, 1985

18. Pasqualini JR: The selective estrogen enzyme modulators in breast cancer: a review. Biochim Biophys Acta 1654: 123-143, 2004.

19. Pasqualini JR and Chetrite GS: Recent insight on the control of enzymes involved in estrogen formation and transformation in human breast cancer. J Steroid Biochem Mol Biol 93: 221-236, 2005.

20. Conley A and Hinshelwood M: Mammalian aromatases. Reproduction 121: 685-695, 2001.

21. Santen RJ and Harvey HA: Use of aromatase inhibitors in breast carcinoma. Endocr Relat Cancer 6: 75-92, 1999.

22. Suzuki T, Miki Y, Nakamura Y, et al: Sex steroid-producing enzymes in human breast cancer. Endocr Relat Cancer 12: 701-720, 2005.

23. Cos S, González A, Álvarez-García V, Alonso-González C and Martínez-Campa C: Melatonin and breast cancer: selective estrogen enzyme modulator. In: Advances in Cancer Drug Targets. Atta-ur-Rahman (ed.) Vol 1. 1st Edition. Bentham Science Publishers, Sharjah (UAE), pp207-237, 2012.

24. González A, Cos S, Martínez-Campa C, Alonso-González C, Sánchez-Mateos S, Mediavilla MD and Sánchez-Barceló EJ: Selective estrogen enzyme modulator actions of melatonin in human breast cancer cells. J Pineal Res 45: 86-92, 2008.

25. Bulun SE, Lin Z, Imir G, et al: Regulation of aromatase expression in estrogen-responsive breast and uterine disease: from bench to treatment. Pharmacol Rev 57: 359-383, 2005.

26. Bulun SE, Sebastian S, Takayama K, Suzuki T, Sasano H and Shozu M: The human CYP19 (aromatase p450) gene: update on physiologic roles and genomic organization of promoters. J Steroid Biochem Mol Biol 86: 219-224, 2003.

27. Díaz-Cruz ES, Shapiro CL and Brueggemeier RW: Cyclooxygenase inhibitors suppress aromatase expression and activity in breast cancer cells. J Clin Endocrinol Metab 90: 2563-2570, 2005.

28. Prosperi JR and Robertson FM: Cyclooxygenase-2 directly regulates gene expression of P450 Cyp19 aromatase promoter regions pII, pI.3 and pI.7 and estradiol production in human breast tumor cells. Prostaglandins Other Lipid Mediat 81: 55-70, 2006.

29. Martínez-CampaC,González A,MediavillaMD,Alonso-González, Álvarez-García V, Sánchez-Barceló EJ and Cos S: Melatonin inhibits aromatase promoter expression by regulating cyclooxygenases expression and activity in breast cancer cells. Br J Cancer 101: 1613-1619, 2009

30. Wang J, Xiao X, Zhang Y, et al: Simultaneous modulation of COX-2, p300, Akt, and Apaf-1 signaling by melatonin to inhibit proliferation and induce apoptosis in breast cancer cells. J Pineal Res 53: 77-90, 2012.

31. González A, Martínez-Campa C, Mediavilla MD Alonso-González C, Sánchez-Barceló EJ and Cos S: Inhibitory effects of pharmacological doses of melatonin on aromatase activity and expresión in rat glioma cells. Br J Cancer 97: 755-760, 2007.

32. González A, Martínez-Campa C, Mediavilla MD, Alonso-González C, Álvarez-García V, Sánchez-Barceló EJ and Cos S: Inhibitory effects of melatonin on sulfatase and $17 \beta$-hydroxysteroid dehydrogenase activity and expression in glioma cells. Oncol Rep 23: 1173-1178, 2010.
33. González A, Álvarez-García V, Martínez-Campa C, Mediavilla MD, Alonso-González C, Sánchez-Barceló EJ and Cos S: In vivo inhibition of the estrogen sulfatase enzyme and growth of DMBA-induced mammary tumors by melatonin. Curr Cancer Drug Tar 10: 279-286, 2010.

34. Meng L, Zhou J, Sasano H, Suzuki T, Zeitoun KM and Bulun SE: Tumor necrosis factor $\alpha$ and interleukin 11 secreted by malignant breast epitelial cells inhibit adipocyte differentiation by selectively down-regulating CCAAT/enhancer binding protein $\alpha$ and peroxisome proliferator-activated receptor $\gamma$ : mechasnism of desmoplastic reaction. Cancer Res 61: 2250-2255, 2001

35. Bulun SE, Chen D, Lu M, et al: Aromatase excess in cancers of breast, endometrium and ovary. J Steroid Biochem Mol Biol 106: 81-96, 2007.

36. Ntambi JM and Kim YC: Adipocyte differentiation and gene expression. J Nutr 130: 3122S-3126S, 2000.

37. González A, Álvarez-García V, Martínez-Campa C, Alonso-González C and Cos S: Melatonin promotes differentiation of 3T3-L1 fibroblasts. J Pineal Res 52: 12-20, 2012.

38. Knower KC, To SQ, Takagi K, et al: Melatonin suppresses aromatase expression and activity in breast cancer associated fibroblasts. Breast Cancer Res Treat 132: 765-771, 2012.

39. Álvarez-García V, González A, Alonso-González C, Martínez-Campa C and Cos S: Melatonin interferes in the desmoplastic reaction in breast cancer by regulating cytokine production. J Pineal Res 52: 282-290, 2012.

40. Harada N, Sasano H, Murakami H, Ohkuma T, Nagura H and Takagi Y: Localized expression of aromatase in human vascular tissues. Circ Res 84: 1285-1291, 1999.

41. Mukherjee TK, Dinh H, Chaudhuri G and Nathan L: Testosterone attenuates expression of vascular cell adhesion molecule-1 by conversion to estradiol by aromatase in endothelial cells: implications in atherosclerosis. Proc Nat Acad Sci USA 6: 4055-4060, 2002.

42. Sebastian S, Takayama K, Shozu M and Bulun SE: Cloning and characterization of a novel endothelial promoter of the human CYP19 (aromatase P450) gene that is upregulated in breast cancer tissue. Mol Endocrinol 10: 2243-2254, 2001.

43. Senger DR, Van De Water L, Brown LF, et al: Vascular permeability factor (VPF, VEGF) in tumor biology. Cancer Metastasis Rev 12: 303-324, 1993.

44. Liang Y and Hyder SM: Proliferation of endothelial and tumor epithelial cells by progestin-induced vascular endothelial growth factor from human breast cancer cells: paracrine and autocrine effects. Endocrinology 146: 3632-3641, 2005.

45. Álvarez-García V, González A, Martínez-Campa C, Alonso-González $\mathrm{C}$ and $\mathrm{Cos} \mathrm{S}$ : Melatonin modulates aromatase activity and expression in endotelial cells. Oncol Rep 29: 2058-2064, 2013.

46. Álvarez-García V, González A, Alonso-González C, Martínez-Campa C and Cos S: Regulation of vascular endotelial growth factor by melatonin in human breast cáncer cells. J Pineal Res 54: 373-380, 2013.

47. Álvarez-García V, González A, Alonso-González C, Martínez-Campa C and Cos S: Antiangiogenic effects of melatonin in endotelial cell cultures. Microvasc Res 87: 25-33, 2013. 